\title{
Development of the spine following pinealectomy or sensorimotor cortical area damage
}

\author{
Richard Chaloupka ${ }^{1}$, Milan Dvořák ${ }^{2}$, František Tichý ${ }^{3}$, Jiří Veselý ${ }^{4}$, Alois Nečas ${ }^{2}$
}

${ }^{1}$ University Hospital, Masaryk University Brno, Faculty of Medicine, Orthopaedic Department, Brno, Czech Republic ${ }^{2}$ University of Veterinary and Pharmaceutical Sciences Brno, Faculty of Veterinary Medicine, Department of Surgery and Orthopaedics, ${ }^{3}$ Department of Anatomy, Histology and Embryology, Brno, Czech Republic ${ }^{4}$ Masaryk University Brno, Faculty of Medicine, Plastic Surgery Department, Brno, Czech Republic

\author{
Received September 30, 2012 \\ Accepted August 28, 2013
}

\begin{abstract}
The aim of this experimental study was to assess the spine development in growing rats following pinealectomy or partial sensorimotor cortical area damage. A total of 68 Wistar albino rats (Rattus norvegicus $v$. alba $f$. domestica) aged 3-4 weeks were divided into four groups. In group $1(\mathrm{n}=22)$ pinealectomy was performed, in group $2(n=24)$ the sensorymotor cortical area $2 \times 1 \times 1 \mathrm{~mm}$ below the coronal suture was removed. Sham operation consisted of a craniotomy $(\mathrm{n}=11)$ and a craniotomy with a durotomy $(n=11)$. All surgeries were performed from the left side. The rats were killed four months after surgery and radiography was then made. Scoliosis, C2-T7 lordosis and T7-S1 kyphosis were measured. The brains of rats after sensorimotor cortical area removal were isolated and investigated including histological examination (light microscope). Scoliosis of 9-14 degrees (mean value 10.8) was developed in five animals after pinealectomy; in rats after removal of the sensorimotor cortical area scoliosis of 10-24 degrees (mean value 15.9) was observed in eight animals. The scoliotic curves were non structural. Our results indicate the importance of cortical area damage, together with craniotomy and durotomy in the development of growing rat spine. These damages could cause a disorder of balance between smaller inhibitory and greater facilitating area of central nervous system, controlling the muscular tone and resulting in the development of increased lordosis and kyphosis and non structural scoliosis due to muscle imbalance. Thus the new hypothesis of scoliosis aetiology was introduced.
\end{abstract}

Idiopathic scoliosis, aetiology, experimental study, pinealectomy, brain damage, rat

The literature presents many hypotheses relating to the aetiology of idiopathic scoliosis. In clinical practice, only the effect of heredity has been confirmed (Gorman et al. 2012; Grauers et al. 2012); in experimental and clinical studies it was the effect of neural dysfunctions localized in some areas of the central nervous system (Machida et al. 1993). Of the all hypotheses on the aetiology of idiopathic scoliosis, the intricacy of the problem follows. Also the results of experimental studies did not contribute to the clarification of the aetiology of the disease (Janssen et al. 2011).

The aim of our experimental study was to evaluate the development of the spine in growing rats after damage of the sensorimotor cortical area.

\section{Materials and Methods}

Animals and experimental design. Ethics Committee Approval was given on September 19, 1997, to conduct the study according to the standard protocol.

In total, 68 Wistar albino rats (Rattus norvegicus v. alba f. domestica) aged 3-4 weeks were divided into 4 groups. In group $1(\mathrm{n}=22$ rats, 7 females, 15 males), pinealectomy (PIN) was performed; in group $2(\mathrm{n}=24,14$ females, 10 males), the sensorimotor cortical area (SMCA) $2 \times 1 \times 1 \mathrm{~mm}$ bellow the coronal suture was removed. Sham operation was conducted in the other two groups ( $\mathrm{n}=11$ for each group) and consisted of a craniotomy (CRA) and a craniotomy with a durotomy (CRDU), respectively. All surgeries were performed from the left side, at an average age of 3.5 weeks with intraperitoneal anaesthesia (diazepam at a $15 \mathrm{mg} / \mathrm{kg}$ dose and $5 \%$ ketamine at a $60 \mathrm{mg} / \mathrm{kg}$ dose). Rats' weight ranged from 40 to $60 \mathrm{~g}$ with the mean weight of $48.6 \mathrm{~g}$. Premedication lasted 5 to $15 \mathrm{~min}$, on average $9.36 \mathrm{~min}$. The operation lasted 6 to $12 \mathrm{~min}$, on the average $7.5 \mathrm{~min}$. After incision of the skin (about $1.5 \mathrm{~cm}$ of length) from the left in the paramedial area from sinus sagittalis sup., at the level of

Address for correspondence:

Doc. MUDr. Richard Chaloupka, CSc.

Orthopaedic Department, University Hospital

Faculty of Medicine, Masaryk University Brno

Jihlavska 20, 62500 Brno, Czech Republic
Phone: +420 532232703

Fax: +420 532233601

E-mail: rchaloup@med.muni.cz

http://actavet.vfu.cz/ 
the bregmatic suture, the $3 \times 2 \mathrm{~mm}$ skull opening was formed with the aid of power drill. After perforation of the dura by injection needle 1 to $2 \mathrm{~mm}$ in length a part of the cortex of the size $2 \times 1 \times 1 \mathrm{~mm}$ was removed from the area closely behind the bregmatic suture with the aid of small forceps, to a depth of approximately $1 \mathrm{~mm}$. The operation was completed by suturing the skin only. The rats were euthanised in an inhalation chamber 4 months after surgery; radiography (posteroanterior and lateral) then followed. Scoliosis, C2-T7 lordosis and T7S1 kyphosis were measured. The brains of rats from SMCA group were isolated, fixed in 10\% formaldehyde, the defect area was evaluated using light microscope (haematoxylin-eosin staining) (Chaloupka 2011).

Statistical analysis

We used ANOVA test for evaluation of potential differences between groups. When approving the difference between groups, we tested the difference between each two groups by two-sample $t$-test. The tests were realized at the 0.05 significance level.

\section{Results}

The rats began awakening approximately 45 to $60 \mathrm{~min}$ after the end of operation. No motor deficits were observed after surgery. In the SMCA group in 4 cases simple curve was found, 3 times the left-sided curve with an apex between T2 - T3, at T13, and between T12 and T13, one right-sided curve with an apex between T13 and L1. In 4 cases it was double curve, 3 times with upper curve larger with an apex between T1 and T2, at T2, and between $\mathrm{T} 2$ and $\mathrm{T} 3$. In one case the curves took an identical value with an apex at T2 and between T13 and L1. In all double curves the upper one was left-sided. The curves were not significant nor structural. The following significant $(P<0.05)$ differences were found: higher surgery weight in PIN, longer surgery time in PIN and SMCA, lower lordosis in PIN and higher in CRDU, differences of all groups in kyphosis and in an end weight. The results of experiments are given in Table 1.

Table 1. Results of four experimental groups in growing rats

\begin{tabular}{lccrr}
\hline Mean values & PIN & SMCA & CRA & CRDU \\
\hline Surgery weight (g) & 69.8 & 48.1 & 50.0 & 48.7 \\
Surgery time (minutes) & 13.5 & 7.5 & 5.8 & 4.8 \\
C2-T7 lordosis (degrees) & -40.1 & -56.2 (from -38 to -73) & -54.6 & -66.0 \\
T7-S1 kyphosis (degrees) & 25.1 & $34.3(19-46)$ & 41.0 & 51.5 \\
End weight (g) & 425.0 & $350.8(260-460)$ & 311.8 & 292.7 \\
Scoliosis (cases, $\%)$ & $5(22.7 \%)$ & $8(33.3 \%)$ & 0 & 0 \\
Curve (degrees) & $10.8(9-14)$ & $15.9(10-24)$ & - & - \\
\hline
\end{tabular}

Experimental groups: PIN - pinealectomy $(n=22)$, SMCA - sensorymotor cortical area damage $(n=24)$, CRA - craniotomy $(\mathrm{n}=11), \mathrm{CRDU}$ - craniotomy and durotomy $(\mathrm{n}=11)$

The range of defects on the frontal lobe was mostly $1-2 \mathrm{~mm}^{2}$; in one rat the defect was rather extensive. In rats with scoliosis the defect was present 4 times in the parietal lobe and 4 times in the frontal lobe. X-rays and histological pictures are demonstrated in Figs 1 and 2 (Plate IV). In other 16 animals the defect was not found once, 10 times it was in the parietal lobe, twice in the frontal lobe, once in the occipital and the temporal lobe, once in the frontal and the parietal lobe simultaneously. In all cases the surface reaction of astroglia with the defect surface reparation was present; only in one rat the reaction was minimal.

\section{Discussion}

Rats achieve the maturity of brain development corresponding to a newborn baby at the age of 10 days; sexual maturation starts in the age of 35 days. At the age of 90 days rats are 
considered adult (Mareš 1991). Scoliosis developed with convexity to the damage side after brain stem damage on rats (Barrios and Arrotegui 1992). Unilateral damage to mesencephalon in chicken embryos resulted in cervical scoliosis in 20 out of 23 cases with concavity on the operated side (McKee and Fergus on 1984). In the highest percentage of cases the origin of scoliosis is described after removal of the corpus pineale. Curves were found in up to $100 \%$ chickens (Machida et al. 1993). Pinealectomy in bipedal rats caused thoracic or thoracolumbar scoliosis in 10 animals. The authors emphasize the disturbance of equilibrium and other postural mechanisms secondary to melatonin deficiency (Machida et al. 2005). In chickens, the origin of scoliosis in high percentage of cases after pinealectomy can be explained by high quantity of melatonin receptors (in the brain stem, in grey medullary mass, especially in the lumbar area) (Wan and Pang 1994). In other animals, these receptors are present in limited quantity in the thalamus, hypothalamus and hypophysis (Utiger 1992). Showing to a small significance of experiments on chickens in the search for aetiology of human idiopathic scoliosis, in addition to the above mentioned data relating to the melatonin receptors, different number of vertebrae and also a different formation of the cortex are emphasized (Novák et al. 1992).

In healthy adult rats the following values were found: cervicothoracic lordosis ranged from $-39^{\circ}$ to $-63^{\circ}$ with a mean value of $-48^{\circ}$, thoracolumbar kyphosis took the values from $+41^{\circ}$ to $+54^{\circ}$, the mean value was $+48^{\circ}$ (Nečas and Dvoŕák 1999). In addition to hypoxia and mechanical damage (cortex), thermal damage (power drill) and also the metabolic-toxic effects from the used anaesthetics may have affected the results of our experiment. We recorded most curves of up to $20^{\circ}$ after Cobb. This finding can be explained by great plasticity of the brain and different damage to the brain. Histological examination proved a defect of the cortex with repairing processes, with the presence of glial cells on the defect surface. Localization of defects in rats with scoliosis, four times in the frontal lobe, corresponds with the motor area. The other 4 cases with damage to the parietal lobe involve damage to the sensory cortex area. In 2 rats with damage to the frontal lobe and in 10 with damage to the parietal lobe scoliosis was not found (Chaloupka 2011).

Cortex involved in the control of the upright position asserts itself during phylogenesis and ontogenesis more and more expressively. The control of posture and balance is secured by a combination of several subcortical systems with a complex set of cortical areas with coordination and cooperation of the reticular formation and cerebellum. The reticular formation is involved in the control of myotatic reflexes, posture reactions, righting reflexes and intentional movements (Novák et al. 1992). Two facilitating areas exist: an extensive area in the reticular formation of the brain stem, and a small in the area of vestibular nuclei. The smaller inhibition area is controlled by the cortex (supressoric zones) and spinal cerebellum. The inhibition areas are located in the cortex, basal ganglia (nucleus subthalamicus and substantia nigra), in the cerebellum and in the small area of the reticular formation.

Subjects with adolescent idiopathic scoliosis showed generalized asymmetry of many brain functions and structures (Goldberg et al. 1995). In children the muscle imbalance is manifested at different stages of development by the rise of scoliosis and lordosis. This balance impairment may be caused by unilateral hereditary disorders of the brain, prenatal damage by different toxins or infections, or perinatal localized ischaemia, followed by the predominance of facilitating areas, controlling dorsal paravertebral muscular tone and body position. Damage can be present in the area of inhibitory centres of the cortex either as a result of evolution anomaly, damage during pregnancy or hypoxia in the perinatal period. Due to the plasticity of the cortex no other symptoms that could be diagnosed by current procedures are present. Damage to the other inhibitory areas can also start up scoliosis followed by great variability of curves and their different progression (Chaloupka 2011). 
Our results indicate the effect of cortical area damage together with the craniotomy and durotomy in the development of the growing rat spine. We cannot exclude the influence of perioperative bleeding, brain hypoxia or metabolic effect of anaesthetics. These damages could cause a disorder of balance in rats between smaller inhibitory and greater facilitating areas of CNS, controling the muscular tone and posture, and resulting in the development of increased lordosis and kyphosis and nonsignificant, nonstructural scoliosis due to muscle imbalance.

\section{Acknowledgments}

The authors would like to thank to Mrs. Milena Černá (Plastic Surgery Department) and Mr. Tomáš Výmola (University of Veterinary and Pharmaceutical Sciences) for their assistance during the experiments.

\section{References}

Barrios C, Arrotegui JI 1992: Experimental kyphoscoliosis induced in rats by selective brain stem damage. SICOT 16: 146-151

Barrios C, Tunon MT, De Salis JA, Beguiristain JL, Canadell J 1987: Scoliosis induced by medullary damage: An experimental study in rabits. Spine 12: 433-439

Chaloupka R 2011: Influence of sensorymotor cortical area damage on spine development - spine development after pinealectomy and sensorymotor area damage. LAP Lambert Academic Publishing. Saarbrücken, Germany, $122 \mathrm{p}$.

Goldberg CJ, Dowling FE, Fogarty EE, Moore DP 1995: Adolescent idiopathic scoliosis and cerebral asymmetry. An examination of a nonspinal perceptual system. Spine 20: 1685-1691

Gorman KF, Julien C, Moreau A 2012: The genetic epidemiology of idiopathic scoliosis. Eur Spine J 21: 19051919

Grauers A, Rahman I, Gerdhem P 2012: Heritability of scoliosis. Eur Spine J 21: 1069-1074

Janssen MM, de Wilde RF, Kouwenhoven JW, Castelein RM 2011: Experimental animal models in scoliosis research: a review of the literature. Spine J 11: $347-358$

Machida M, Dubousset J, Imamura Y, Iwaya T, Yamada T, Kimura J 1993: An experimental study in chickens for the pathogenesis of idiopathic scoliosis. Spine 18: 1609-1615

Machida M, Saito M, Dubousset J, Yamada T, Kimura J, Shibasaki K 2005: Pathological mechanism of idiopathic scoliosis: experimental scoliosis in pinealectomized rats. Eur Spine J 14: 843-848

Mareš P 1991: Epileptic phenomena in the immature brain. Physiol Res 40: 577-584

McKee GJ, Ferguson MWJ 1984: The effects of mesencephalic neural crest cell extirpation on the development of chicken embryos. J Anat 139: 491-512

Nečas A, Dvořák M 1999: Development of the spine following pinealectomy in growing rats. Acta Vet Brno 68: 31-39

Novák M, Faber J, Kufudaki O 1992. Neural networks and information systems of living organisms. (in Czech) Grada, Praha

Utiger RD 1992: Melatonin: The hormone of darkness. New Eng J Med 327: 1377- 1379

Wan QI, Pang SF 1994: Segmental, coronal and subcellular distribution of 2- (125I) iodo melatonin binding sites in the chicken spinal cord. Neurosci Lett 180: 253-256 
Plate IV

Chaloupka R. et al.: Development of ... pp. 411-414

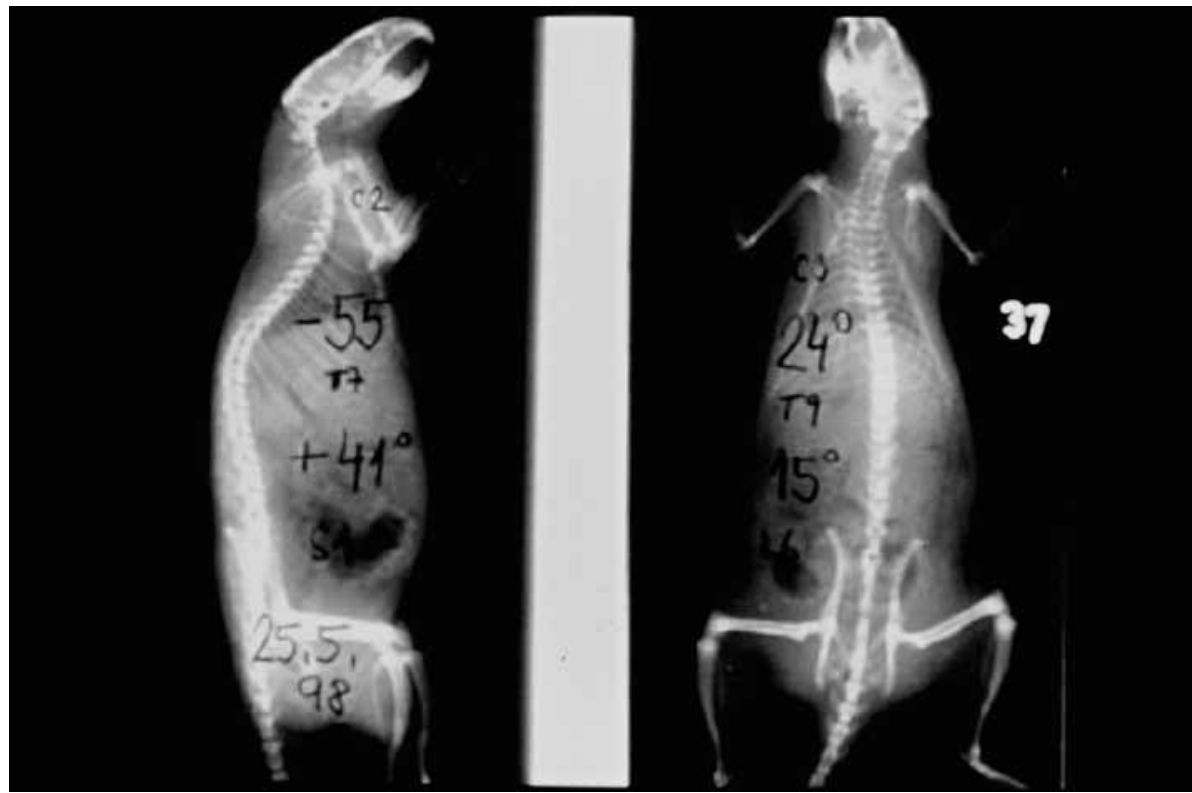

Fig. 1. X-rays of a rat (No. 37) four months after pinealectomy (Chaloupka 2011).

Double curve, the upper one with apex between T2 and T3, C3 $24^{\circ} \mathrm{T} 915^{\circ} \mathrm{L} 6$, sagittal plane $\mathrm{C} 2-55^{\circ} \mathrm{T} 7$ $+41^{\circ} \mathrm{S} 1$.

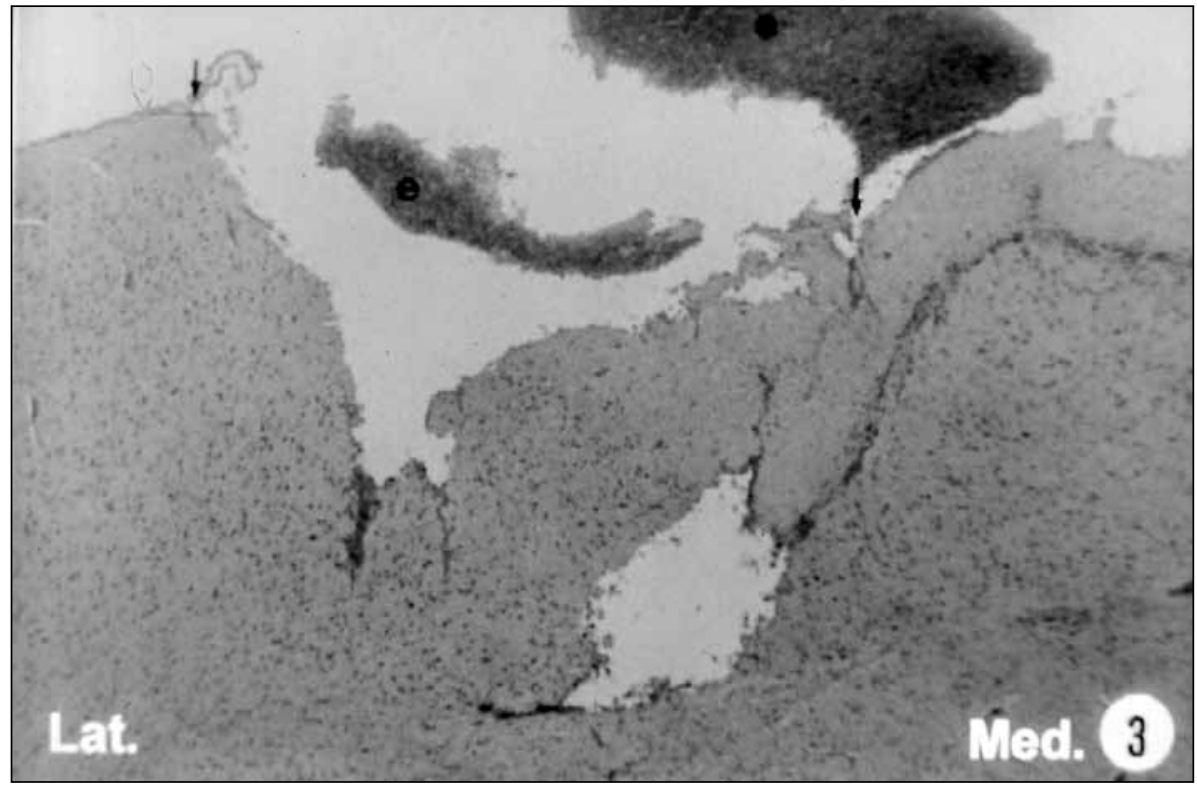

Fig. 2. Histological picture of the brain of a rat (No. 37) after pinealectomy (Chaloupka 2011).

Section in the frontal plane. Defect in the frontal lobe of the left hemisphere dorsally (between arrows), $2.5 \times 1 \mathrm{~mm}$. It goes up to medullary lamella, locally, up to into it. All six cortical layers are damaged, clusters of erythrocytes present in the defect and its enviroment (e). Stained by haematoxylin-eosin (HE). Magnified $\times 60$. 